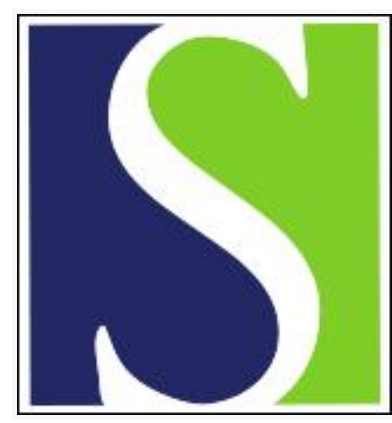

Scand J Work Environ Health 1979;5(3):262-270

https://doi.org/10.5271/sjweh.3100

Issue date: Sep 1979

The transmittance and absorption properties of contact lenses by Nilsson SEG, Lövsund P, Öberg PÅ, Flordahl L-E

Affiliation: Department of Ophthalmology, University Hospital, University of Linkoping, S-581 85 Linkoping, Sweden.

Refers to the following text of the Journal: 1979;5(3):271-279

The following articles refer to this text: 1979;5(3):271-279; 1979;5(3):280-285

Key terms: absorption; absorption property; contact lense; radiation hazard; spectrophotometry; transmittance

This article in PubMed: www.ncbi.nlm.nih.gov/pubmed/20120574

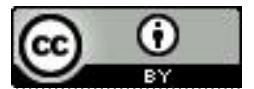




\title{
The transmittance and absorption properties of contact lenses
}

\author{
by SVEN ERIK G. NILSSON, M.D., Ph.D., ${ }^{1}$ PER LÖVSUND, M.Sc.E.E., ${ }^{2}$
}

P. ÅKE ÖBERG, Ph.D., ${ }^{2}$ and LARS-ERIK FLORDAHL, M.Sc. ${ }^{3}$

\begin{abstract}
NILSSON, S. E. G., LÖVSUND, P., ÖBERG, P. A. and FLORDAHL, L.-E. The transmittance and absorption properties of contact lenses. Scand. j. work environ. \& health 5 (1979) 262-270. The transmittance and absorption properties of certain soft-lens materials with a low and a high water content and a hard-lens material were analyzed by a spectrophotometric technique over a broad spectrum from ultraviolet $(195 \mathrm{~nm})$ to far infrared $(40,000 \mathrm{~nm})$ radiation. The dry lens materials not only showed marked absorption within the short-wave region of ultraviolet, but also very strong absorption within the infrared range, especially medium and far infrared. Wet soft-lens material showed greatly increased absorption within the infrared regions in which water shows absorption maximums. Therefore the wet softlens materials absorbed nearly $100 \%$ of the radiation within the greater part of the medium and far infrared ranges. Transmittance and absorption were inevitably affected by the thickness of the material, but differences between the various makes of lens were minor. It is clear that sources of high-energy radiation, particularly within the infrared spectra, may constitute hazards with regard to the absorption of radiation and the consequent generation of heat in contact lenses.
\end{abstract}

Key words: absorption, contact lenses, radiation hazards, spectrophotometry, transmittance.

Repeated warnings have appeared in recent years about the wearing of contact lenses in connection with welding and other electric arcs $(1,7,11,13,14,17)$. The start seems to have been the case of a shipyard worker with contact lenses who, in spite of using safety glasses, developed corneal lesions after exposure to a power-

1 Department of Ophthalmology, University of Linköping, Sweden.

2 Department of Biomedical Engineering, University of Linköping, Sweden.

3 Institute of Optical Research, Stockholm, Sweden.

Reprint requests to: Prof. S. E. G. Nilsson, Department of Ophthalmology, University Hospital, University of Linköping, S-581 85 Linköping, Sweden. ful flash that occurred when the circuit breaker arced during the connection of a welding cable. When the man later removed his lenses, large areas of dried cornea were reported to have come away with them. (Probably only the superficial epithelial layer was involved.) The explanation that was suggested at the time was that the contact lenses had in some way concentrated the heat from the flash to the cornea and thus injured it $(13,14)$. It was later found that the lenses had been worn continuously for $17-18 \mathrm{~h}$, and this explanation was regarded by some as sufficient for the drying and separation of the epithelium when the lenses were taken out $(11,14,15,16)$. The incident has recently been described as an "industrial atrocity story" (16). 
We have been unable to find any experimental evidence to support the claims that have been made, however, and we therefore feel it is a matter of some urgency to define the absorption properties of different contact lens materials so that possible hazards can be assessed. Little data on these matters have been published. The major works $(3,4)$ on infrared absorption by polymers give relevant details on certain individual substances, but the contact lens materials on the market today commonly consist of different combinations of polymers, chiefly acrylates and vinyl copolymers. Furthermore, the detailed composition of the material is frequently kept secret. It is therefore important to measure the actual lens material in thicknesses not greatly different from those in clinical use. Highgate (2) has published a graph comparing the transmittance in water, polymethyl methacrylate (the material in hard lenses), and glass, but we have been unable to find detailed analyses of soft-lens materials.

The work presented in this report is concerned with the transmittance and absorption properties of contact lens materials examined by a spectrophotometric technique over a broad region of the spectrum from short-wave ultraviolet to far infrared. Our objectives were to establish whether contact lenses absorb electromagnetic radiation to a dangerous degree, to ascertain the parts of the spectrum to which the greater part of the absorption is confined, and to investigate whether significant differences in these respects exist between different makes of lens and different materials. The absorption by the fluid in the lenses was also taken into account.

Two other studies have been in progress parallel to this one, namely, analyses of temperature changes in contact lenses (free-hanging and in situ on rabbit eyes) in connection with radiation from certain welding processes (5) and from infrared heaters (6). Surprisingly large temperature increases have been found in the lenses in comparison with the environmental conditions.

\section{MATERIAL AND METHODS}

Transmittance measurements were performed on eight different objects, specified in detail in table 1 . They were one test plate (parallel-plane) of material for hard contact lenses (polymethyl methacrylate, PMMA), and three test plates and four plano lenses ( \pm 0 diopters) of material for soft lenses (polyhydroxyethyl methacrylate, HEMA). Other constituents of the lens materials are not fully known, as the manufacturers have not disclosed the compositions, but there is no doubt that the soft lenses also contain vinyl copolymers. Two of the plano lenses had a high water content $(85 \%)$, and the remaining materials a low water content $(39 \%)$. In this paper the test plates and plano lenses will be referred to as "material" or "lens material."

Materials I-VIII were analyzed after being dried in air (at least $24 \mathrm{~h}$ at room temperature in special instruments to maintain the original radius of curvature

Table 1. Plano lenses and test plates of contact lens material used for the measurement of transmittance.

\begin{tabular}{lllllll}
\hline Test object & Manufacturer & Material & $\begin{array}{c}\text { Water con- } \\
\text { tent }(\%)\end{array}$ & $\begin{array}{c}\text { Type of } \\
\text { object }\end{array}$ & $\begin{array}{c}\text { Thickness, } \\
\text { dry (mm) }\end{array}$ & $\begin{array}{c}\text { Thickness, } \\
\text { wet (mm) }\end{array}$ \\
\hline I & Hydron & HEMA & 39 (soft) & Plano lens & 0.5 & 0.6 \\
II & Hydron & HEMA & 39 (soft) & Test plate & 0.7 & 0.8 \\
III & Weicon & HEMA & 39 (soft) & Plano lens & 0.5 & 0.6 \\
IV & Wöhlk & HEMA & 39 (soft) & Test plate & 0.7 & 0.8 \\
V & Soflens & HEMA & 39 (soft) & Test plate & 0.7 & 0.8 \\
VI & Scanlens & HEMA & 85 (soft) & Plano lens & 0.3 & 0.5 \\
VII & Scanlens & HEMA & 85 (soft) & Plano lens & 0.4 & 0.7 \\
VIII & Scanlens & PMMA & c. 1-2 (hard) & Test plate & 0.7 & 0.7 \\
\hline
\end{tabular}


and the smoothness of the surface) and thereafter I and VIII were placed in a vacuum $(24 \mathrm{~h}$ at room temperature to a final vacuum of $3 \cdot 10^{-4} \mathrm{~N} / \mathrm{m}^{2}$ ) and I, VI, and VIII were wet with physiological saline. The edges of all the wet materials were dried in such a way that the surfaces, including the concave surface of the plano lenses, showed no visible excess of fluid. During the measurements, both the dry and wet materials were applied to an aperture with a free access of air to both sides. Measurements in cells proved futile, since the large amount of fluid gave an extremely high absorption of infrared. Soft lens I was $56 \%$ heavier in the wet than in the dry state. Vacuum drying removed fluid corresponding to $3 \%$ of the weight in the air-dried state.

The different measuring regions are defined as follows: ultraviolet (UV) 195-390 $\mathrm{nm}$, visible (VIS) $390-750 \mathrm{~nm}$, near infrared (NIR) $750-2,700 \mathrm{~nm}$, medium infrared (MIR) 2,700-14,000, and far infrared (FIR) $14,000-40,000 \mathrm{~nm}$.

Transmittance within the UV, VIS and NIR regions was measured in a PerkinElmer model 350 spectrophotometer (Perkin-Elmer Corp., CT, U.S.A.). The MIR and FIR measurements were carried out with a Beckman type IR 20 A spectrophotometer (Beckman Instruments, Inc., CA, U.S.A.).

For the UV measurements the object was applied over an aperture $10 \mathrm{~mm}$ in diameter, centered in a fixed position 67 $\mathrm{mm}$ in front of the detector. The $100 \%$ transmittance level was set with the aperture in place but with no object. The surface of each lens material to be measured was about $79 \mathrm{~mm}^{2}$ (corresponding to the diameter of the aperture), and the angle of aperture was $19^{\circ}$ towards the detector.

For the VIS and NIR measurements a microattachment with diffusing paper was

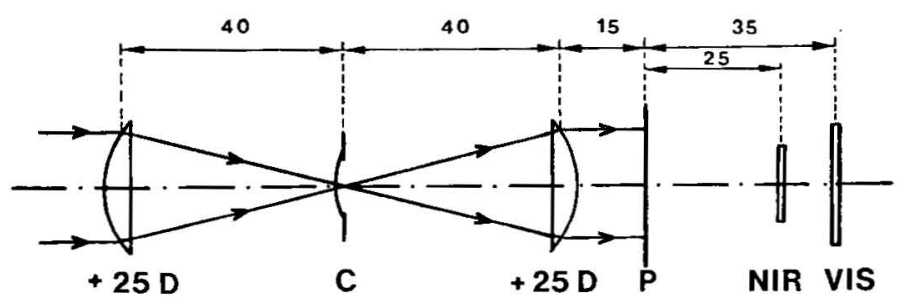

used, the paper being placed 35 and $25 \mathrm{~mm}$, respectively, in front of the detector (fig. 1). This attachment was employed so that all light available in the spectrophotometer would be used and the risk of measurement errors due to the scattering of light caused by possible surface irregularities in the material reduced. It consisted of two positive lenses with a focal distance of $40 \mathrm{~mm}$. The lens material was placed over an aperture in the focal plane of the two lenses. The central area to be tested was about $3 \mathrm{~mm}^{2}$. The angle of the aperture was $33^{\circ}$ towards the detector. The $100 \%$ transmittance level was set with the microattachment in situ in the beam but with no object.

For the MIR and FIR measurements the object was centered over an aperture 10 $\mathrm{mm}$ in diameter. The area to be tested was about $3 \times 6 \mathrm{~mm}$, i.e., about $18 \mathrm{~mm}^{2}$, and the angle of aperture was $6 \times$ $12^{\circ}$ towards the detector. The $100 \%$ transmittance level was set with the aperture in situ.

With limited angles of aperture, diffuse light scatter may constitute a source of error in transmittance measurements of this type. Within the UV, VIS, and NIR ranges, however, the error was probably less than $5 \%$, and within the MIR and FIR spectra not more than $10 \%$. It should also be noted that the sensitivity of the instrument does not permit definite conclusions with regard to the appearance and levels of the curves within the MIR and FIR regions for transmittances of less than $5 \%$. Since the absorption is calculated from the transmittance readings, the possibility of certain losses due to reflection must be borne in mind. These are of the order of $2-4 \%$ per surface, and
Fig. 1. Microattachment and beam path in measurements of the transmittance by contact lens material within the visible and near infrared regions. $(\mathrm{C}=$ contact lens material applied over a 10-mm aperture, $\mathrm{P}=$ diffusing paper, NIR = arrangement of detector for measurements within the near infrared region, VIS = arrangement of detector for measurements within the visible spectrum) (Distances in millimeters) 
they are the least with the shortest wavelengths. In this particular connection the loss due to reflection, including diffuse reflection, was probably not more than about $10 \%$.

The measurements showed extremely good reproducibility. The wet materials were measured twice in succession in the NIR region without rewetting; the curve showed only a change of about $1 \%$. Remeasurement after rewetting gave the same close agreement. This experiment showed, in addition, that during the course of the experiment the materials did not dry out enough to influence the measurements.

\section{RESULTS}

\section{"Dry" lens materials}

Ultraviolet region. In the ultraviolet spectrum most of the materials (I, II, IV-VIII) started transmitting at $245-250 \mathrm{~nm}$, and the curves then climbed sharply (I, II, IV), less sharply (V, VIII), or moderately (VI, VII) before leveling off towards the region of visible light (fig. 2-5). Material III did not start to transmit more than a few percent before about $280-290 \mathrm{~nm}$, but the curve then rose sharply (fig. 3). (Lens material IV is not illustrated in the diagram; it transmitted about 10-20\% more than material II within the greater part of the UV range and about $5 \%$ more within the VIS and the first part of the NIR region. From approximately $1,300 \mathrm{~nm}$ up, however, these two materials were practically identical.)

Visible region. The various materials differed insignificantly in the VIS region (fig. 2-5). In the dry state the materials with a high water content (VI, VII) showed the least transmittance. At $390 \mathrm{~nm}$ the transmittance varied between approximately $73 \%$ (VII) and about $90 \%$ (I), and at $750 \mathrm{~nm}$ between approximately $84 \%$ (VII) and about $92 \%$ (VIII).

Near infrared region. Within the NIR region nearest visible light all soft materials behaved in a fairly similar manner, the transmittance being almost identical to that for the VIS range. As the wavelength increased, the transmittance successively diminished, and the various materials showed striking differences (fig. 24). Specific absorption peaks occurred at about 1,430, 1,700, 1,920, 2,100, 2,270, and $2,470 \mathrm{~nm}$. After vacuum drying for $24 \mathrm{~h}$ to a final vacuum average of $3 \cdot 10^{-4} \mathrm{~N} /$ $\mathrm{m}^{2}$, lens material I showed reduced absorption at $1,920 \mathrm{~nm}$ from about 51 to $34 \%$. In other respects the absorption remained practically unchanged.

Comparison between the various softlens materials within the upper part of this region showed transmittance to be related to the thickness of the material (fig. 2-4), in contrast to the findings in the UV region. From 2,250 to $2,700 \mathrm{~nm}$ transmittance varied between as little as about 5 and $20 \%$ for the thickest materials (II, IV, V), between about 10 and $35 \%$ for the medium-thick materials (I, III), and between about 25 and $70 \%$ for the thinnest materials (VI, VII). Absorption was thus sometimes considerable.

The hard material (VIII) showed some divergency from the transmittance patterns of the other materials, chiefly because the absorption peaks at 1,430 and $1,920 \mathrm{~nm}$ were much lower and because transmittance increased fairly sharply from 2,400 to $2,700 \mathrm{~nm}$ (fig. 5). On the whole, material VIII resembled material I the closest.

Medium and far infrared regions. The transmittance patterns of all the soft materials were similar in the MIR and FIR regions. They showed a maximum basic transmission of $5-10 \%$ interrupted by certain peaks, chiefly within the region of $3,500-5,700 \mathrm{~nm}$, where the transmittance decreased with increasing thickness of the materials from approximately $55 \%$ (material VI) to $5-10 \%$ (material V) (fig. 2-4). Furthermore, at least the thinnest material (VI) showed distinct peaks at about 3,200 and $6,500 \mathrm{~nm}$, and smaller peaks within the region of approximately $9,000-15,000 \mathrm{~nm}$.

The hard material (VIII) (fig. 5) did not differ greatly from the soft. Several moderate peaks were present between 3,500 and $5,700 \mathrm{~nm}$. A few extra peaks were present in the beginning of the MIR 